\title{
FEA-BASED STRUCTURAL HEAT TRANSFER CHARACTERISTIC OF 3-D ORTHOGONAL WOVEN COMPOSITE SUBJECTED TO THE NON-UNIFORM HEAT LOAD
}

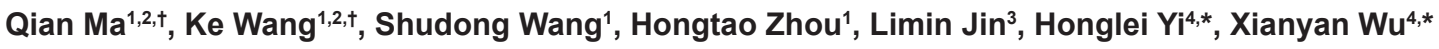 \\ 1 Department of Textile and Clothing, Jiangsu Research and Development Center of the Ecological Textile Engineering and Technology, \\ Yancheng Polytechnic College, Yancheng, 224005, China \\ 2 Engineering Research Center for Clean Production of Textile Printing and Dyeing, Ministry of Education, Wuhan, 430073, China \\ 3 Shanghai Institute of Applied Physics, Chinese Academy of Sciences, Shanghai, 201204, China \\ 4 Key Laboratory of Yarn Materials Forming and Composite Processing Technology of Zhejiang Province, College of Material and Textile Engineering, \\ Jiaxing University, Jiaxing, 314001, China \\ *Corresponding authors. E-mail: Honglei Yi (yi-hl@163.com); Xianyan Wu (xywu@zjxu.edu.cn) \\ ${ }^{\dagger}$ These authors have contributed equally to this work.
}

\begin{abstract}
:
The thermodynamic behavior of 3-D orthogonal woven composite is studied to explore its structural heat transfer mechanism in a non-uniform heat load field based on finite element analysis (FEA). The temperature distribution characteristics of the resin matrix and the fabric reinforcement are observed to compare the heat absorption. Furthermore, the dynamic expansion and distribution characteristics of temperature in the 3-D orthogonal woven composite structure have also been quantitatively studied, together with simultaneously obtaining the path characteristics of the heat transfer in each system (i.e., warp yarns, weft yarns, and Z-yarns). In addition, the spatial temperature distribution characteristics of each yarn system in the fabric reinforcement are also explored. Thus, the structural mechanism of heat conduction for 3-D orthogonal woven composite is obtained.
\end{abstract}

\section{Keywords:}

3-D orthogonal woven composite; heat transfer; non-uniform heat load; finite element analysis

\section{Introduction}

In recent decades, textile structural fabrics and their reinforced composite materials have been increasingly widely used in engineering due to their good mechanical properties, such as light weight and high strength. More attention has been paid to the category of this kind of materials, especially for the excellent three-dimensional (3-D) textile structural materials. The most significant feature of the $3-D$ textile structural fabrics is the presence of buckling and undulating yarns in the thickness direction, which makes the yarns between the adjacent layers more closely, enhances the overall loadbearing capacity of the materials, and effectively improves the mechanical performances. Therefore, this type of materials can effectively avoid the occurrence of delamination failure and enhance the damage tolerance. Using the specific processing techniques, such as vacuum-assisted resin transfer molding method (VARTM), winding method, pultrusion process, etc., the 3-D fabrics and resin can be used as the reinforcements and matrix, respectively, to manufacture the resin-based 3-D fabric reinforced composite materials, whose mechanical properties are significantly better than those of the 2-D composite materials. Since the main load-bearing parts of the composite materials are the reinforcements-that is, since the main mechanical properties of the composite materials are determined by the performance of the fabric reinforcementsthe resin-based 3-D fabric reinforced composites have excellent mechanical properties, and this type of materials has been widely used in aviation aerospace, sports equipments, personal protection, transportation, and other fields [1-6].

In the field of engineering applications, composite materials will be affected not only by external mechanical loads but also by external heat load, such as the composite fan blades that have been long-term applied in high-temperature areas. In the atmospheric environment, the intrusion of heat ensures that the thermodynamic properties of composite materials are rigorously tested, and the long-term thermal-oxygen coupling effect leads to the thermal oxidative aging damage of composite materials $[7,8]$. Besides, the heat is gradually transferred to the inside of the material structure through the resin matrix and fiber reinforcement, thereby generating a certain amount of thermal deformation and thermal stress. In particular, due to the difference in thermal conductivity and thermal expansion between the matrix and the fiber-reinforced body, it is easy to cause the debonding phenomenon at the interfaces between the fibers and resin, and the internal micro-cracks are also experience the initialization and propagation stages, which will inevitably greatly affect the overall mechanical properties of the composite materials $[9,10]$. Therefore, studying the temperature distribution effects of the fabric-based composites in the thermal environment has important engineering significance for understanding the temperature expansion path 
and damage mechanism of the composite materials in the thermal fields.

At present, there are related studies on the mechanical damage of the resin-based 3-D textile structural composites in thermal fields [11-13]. Nevertheless, there is still a lack of in-depth study on the structural heat transfer mechanism of the 3-D orthogonal woven composites under the non-uniform thermal load. This observation holds good especially for studies dealing with the spatial temperature distribution characteristics of the main components (fiber reinforcement and resin matrix) of the fiber-reinforced composite materials in the non-uniform thermal load environment, which is of great significance for revealing the thermodynamic properties and damage mechanism of the composite materials in this category.

Furthermore, the thermodynamic behavior of composite materials under specific thermal load, conditions can be explored through the experimental analysis method. However, due to the limitations of experimental and analytical conditions, some information cannot be effectively characterized by experimental approaches, but the information is essential for in-depth understanding of the thermodynamic response and failure mechanism of composite materials. With the rapid development of computer-aided design technology, the finite element analysis (FEA) method provides a possibility to solve this problem. Thus, FEA can be employed to analyze and predict the thermodynamic behavior of fiber-reinforced composites that are subjected to heat loads. FEA has become a very powerful tool in the field of thermodynamic structure design of composites [14-18].

In this paper, the thermodynamic behavior of 3-D orthogonal woven composite is studied to explore its structural heat transfer mechanism in a non-uniform heat load field based on FEA. The temperature distribution characteristics of the resin matrix and the fabric reinforcement are observed to compare the heat absorption. Furthermore, the dynamic expansion and distribution characteristics of temperature in the 3-D orthogonal woven composite structure have also been quantitatively studied, and simultaneously the path characteristics of the heat transfer in each system (i.e., warp yarns, weft yarns, and Z-yarns) are obtained. In addition, the spatial temperature distribution characteristics of each yarn system in the fabric reinforcement are also explored. Thus, the

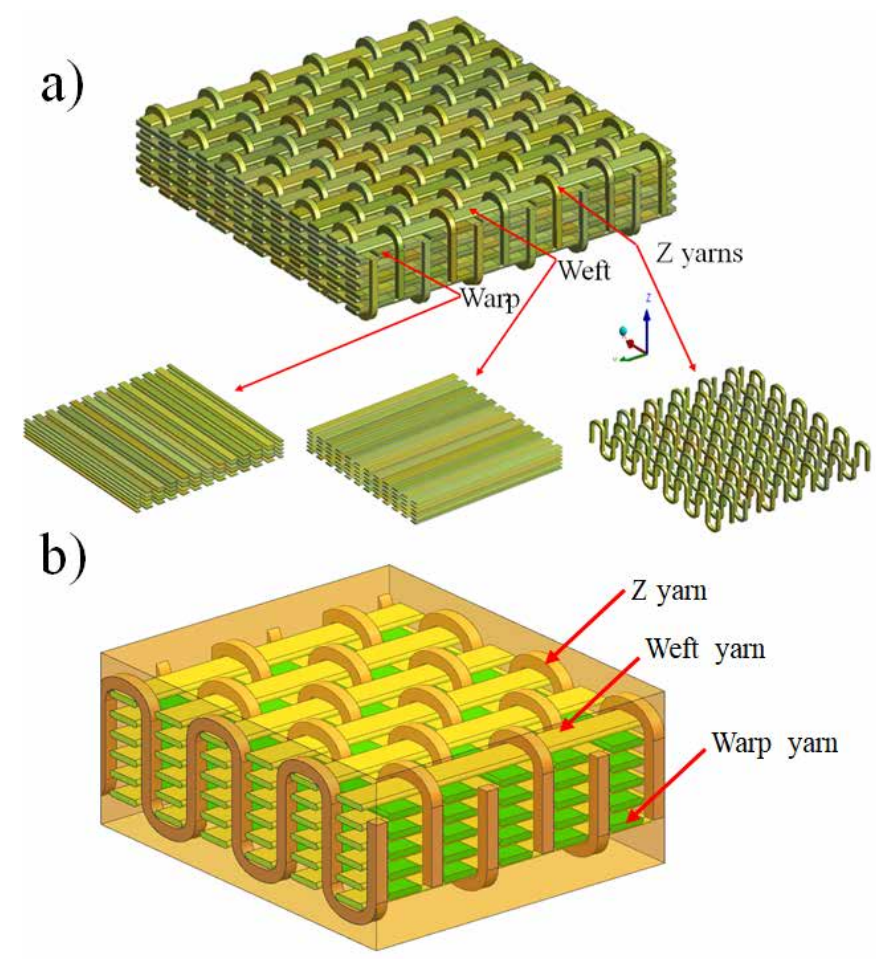

Figure 1. The structures of 3-D orthogonal woven fabric and composite.

structural mechanism of heat conduction for 3-D orthogonal woven composite is obtained.

\section{Materials and FEA models}

\subsection{Materials}

Figure 1 (a) shows the typical structure of the 3-D orthogonal woven fabric. It can be seen that the buckling and undulating Z-yarn system runs through the entire thickness of the material and introduces the binding effect for the warp and weft yarn systems, which are arranged at an angle of $90^{\circ}$ to each other, thereby forming a complete 3-D fabric structure with good structural stability.

In addition, the 3-D orthogonal woven composite can be formed with the epoxy resin, as shown in Figure 1 (b). The raw material of the 3-D orthogonal woven fabric is glass fiber. The material parameters of yarns and resin are listed in Table 1.

Table 1. Material parameters of the yarns and resin.

\begin{tabular}{|c|c|c|c|c|c|c|}
\hline Part & Material & Density $/ \mathbf{g} \cdot \mathrm{cm}^{-3}$ & $\begin{array}{c}\text { Elastic } \\
\text { Modulus/GPa } \\
( \pm 5 \%)\end{array}$ & $\begin{array}{l}\text { Poisson's } \\
\text { ratio }\end{array}$ & $\begin{array}{c}\text { Thermal } \\
\text { Conductivity/W } \cdot \mathrm{m}^{-1} \cdot \mathrm{K}^{-1} \\
( \pm 2 \%)\end{array}$ & $\begin{array}{l}\text { Thermal } \\
\text { radiation } \\
\text { coefficient }\end{array}$ \\
\hline $\begin{array}{c}\text { Warp/Weft } \\
\text { yarn }\end{array}$ & $\begin{array}{l}\text { Glass } \\
\text { fiber }\end{array}$ & 2.50 & 70.00 & 0.20 & 1.30 & 0.8 \\
\hline Z-yarn & $\begin{array}{l}\text { Glass } \\
\text { fiber }\end{array}$ & 2.50 & 65.00 & 0.22 & 1.30 & 0.8 \\
\hline Matrix & $\begin{array}{l}\text { Epoxy } \\
\text { resin }\end{array}$ & 1.05 & 3.65 & 0.40 & 0.35 & 0.9 \\
\hline
\end{tabular}




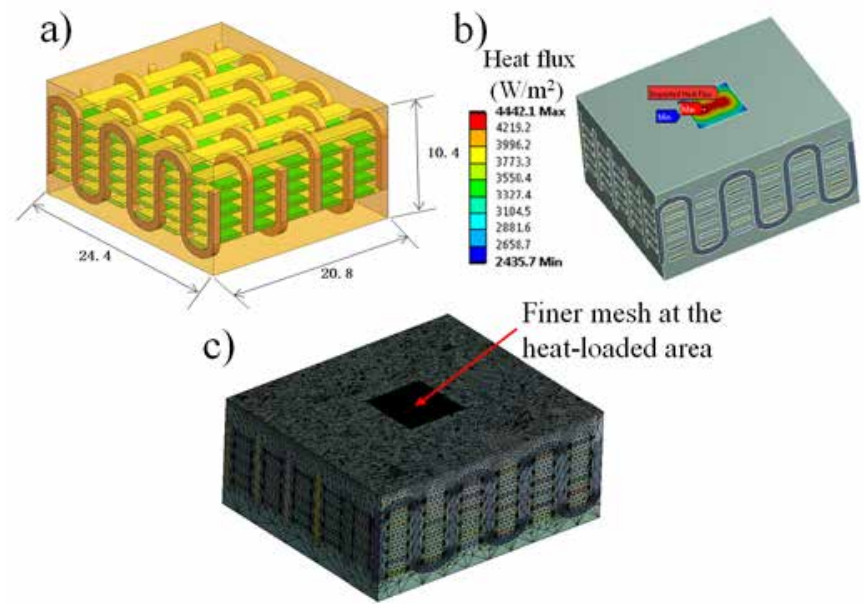

Figure 2. Finite element model of the 3-D orthogonal woven composite subjected to the non-uniform heat load: (a) the model (unit: $\mathrm{mm}$ ); (b) loading and boundary conditions; (c) mesh scheme (coincidence of nodes).

\subsection{Finite element model}

The "Steady-state thermal" analysis module of the commercial finite element software ANSYS ${ }^{\circledR}$, and Windows XP 32-bit version operating system, are used for FEA.

Figure 2 (a) shows the established finite element model of the heat conduction system of the 3-D orthogonal woven composite. Table 2 lists the relevant parameters of each component in the model.

The contact, load, boundary conditions, and meshing settings for the finite element model are described below.

\section{Contact conditions}

In the model, the contact thermal resistance between the adjacent systems is not considered, and the contact condition between the resin and each yarn system is set to "bonded" contact.

\section{Load and boundary conditions}

Figure 2 (b) shows the load and boundary conditions of the model. A small amount of non-uniformly distributed thermal load is applied to the central area of the upper surface of the composite material, thereby transferring heat to each component through the thermal conductivity of the medium. In

Table 2. The related parameters of the parts in the established finite element model.

\begin{tabular}{|c|c|c|}
\hline System & Number of layers & number \\
\hline Warp yarns & 5 & 25 \\
\hline Weft yarns & 6 & 36 \\
\hline Z-yarns & 1 & 6 \\
\hline Matrix & - & 1 \\
\hline
\end{tabular}

addition, heat dissipation is carried out by the heat radiation effect of the different component surfaces.

\section{Meshing}

The meshing result of the system is shown in Figure 2 (c). For warp, weft, and Z-yarns with the regular geometric shapes, the mesh is divided into hexahedral-based units. For the resin matrix, the tetrahedron is used to divide the mesh. In particular, for the heat-loaded area at the center of the composite surface, a significantly finer mesh is applied. The number of elements for warp yarn system, weft yarn system, Z-yarn system, and resin are $172480,205920,46158$, and 3918, respectively.

\section{Results and discussions}

\subsection{Temperature distribution}

After the resin-based textile structural composite is subjected to an external heat load, the heat first impacts the central area of the resin surface, and then the heat is transferred to the surrounding area along the transverse and longitudinal directions, respectively. In order to produce a more detailed summary of the heat transfer structural effect of the 3-D orthogonal woven composite under a kind of specific nonuniformly distributed heat load, it is necessary to explain the functions of resin, warp, weft, and Z-yarns. Figure 3 shows the temperature distribution of the above-mentioned components after the entire system reaches the steady state.

\section{(1) Resin}

Figure 3 (a) shows the temperature distribution on the resin matrix. It can be found that the temperature on the surface of the resin is the highest due to the direct action area of the heat load, and the highest temperature in the central area reaches $74{ }^{\circ} \mathrm{C}$. In addition, the distribution law of the temperature gradient is consistent with the distribution state of the heat load.

\section{(2) Fabric reinforcement}

The temperature distribution of the fabric reinforcement is shown in Figure 3 (b). Since the absorbed heat of the fabric reinforcement is first transferred from the resin, its maximum

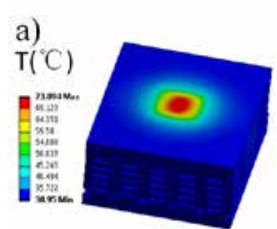

d)

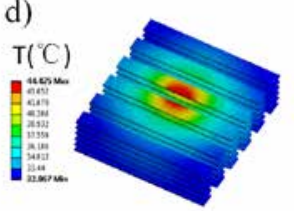

b)

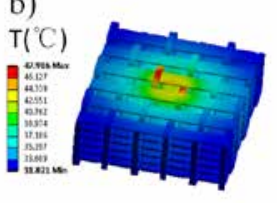

e)

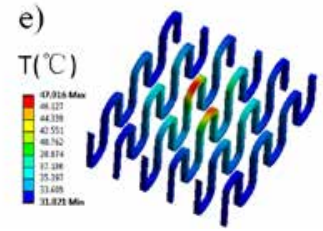

Figure 3. Temperature distribution: (a) resin; (b) fabric; (c) warp yarns; (d) weft yarns; and (c) Z-yarns. 
temperature is significantly lower than that of the resin, approximately $48{ }^{\circ} \mathrm{C}$, which is about $65 \%$ of the maximum temperature in the resin. Therefore, considering that the heat resistance performance of the resin is much lower than that of the glass fibers, the heat-induced damage of the composite material may have first germinated from the resin. Then it mainly propagates to the internal structure along the interface between the resin and the fibers, and finally reaches the fiber reinforcement.

Furthermore, it can be observed that the highest temperature appears on the undulating Z-yarns. Since the central Z-yarn is located closest to the resin, it is the first part to be impacted by heat load, especially at the location near the direct action area of the heat load. In particular, Figure 3 (c), (d), and (e) show the respective temperature distribution characteristics of the warp yarn system, the weft yarn system, and the Z-yarn system, respectively. It can be observed that, as mentioned above, the Z-yarn system has the highest temperature, which is followed by the weft yarn system, at approximately $44^{\circ} \mathrm{C}$, and then the warp yarn system, which has the lowest temperature, whose maximum value is approximately $42{ }^{\circ} \mathrm{C}$. It is significant that the result is closely related to the structural characteristics of the 3-D orthogonal woven fabric reinforcement of the composite.

In particular, because the Z-yarns are relatively longer and undulating, and this state runs through the entire thickness direction of the material structure, the high-temperature zone of each Z-yarn generally exists in the central area only, and the range is relatively narrow. It is difficult for the heat to propagate along the length direction. Thus, the temperature at both ends is lower. The thermal damage is generally limited to the central high-temperature area. Nevertheless, for warp and weft yarns, the situation is significantly different. Since the warp and weft yarns are arranged in a straight way without buckling, their length is relatively shorter. The central high temperature decreases steadily to the two ends, and the range is relatively wide. The low temperature at both ends is a little higher than that of the adjacent Z-yarn. Therefore, the thermal damage can easily germinate from the central area, and propagates to both ends.

\subsection{Temperature distribution path characteristics}

In this sub-section, we explain the procedure adopted to further quantitatively explain the dynamic expansion and distribution characteristics of heat in the 3-D orthogonal woven composite structure, and then obtain the heat transfer mechanism. As shown in Figure 4 (a), we take the area where the heat load is directly applied as the center, and take the warp, weft, and thickness directions to investigate the temperature distribution in the above three paths, and the temperature distribution curve of each path is obtained, as shown in Figure 4 (b).

The heat distribution characteristics of the composite can be observed when subjected to an external heat load. First, for the two directions of transverse warp and weft, the temperature distribution and change trend on these two paths are almost the same - that is, the temperature at the center of the heat load is the highest, and drops sharply in the two directions to the

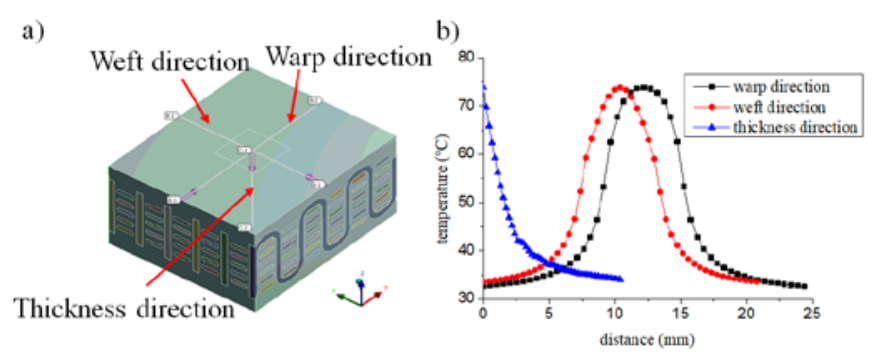

Figure 4. (a) The specific paths; (b) the temperature distribution curves on the specific paths.

ends; besides, the descending speed slows down at the ends. The temperature drops from the highest at the center, where it is in the range of about $74^{\circ}-33^{\circ} \mathrm{C}$; the drop is approximately $55 \%$. Similarly, in the thickness direction, the temperature change trend is similar to those in the warp and weft directions - that is, the temperature at the starting point is the highest, and it drops sharply along the thickness direction, with a drop of approximately $55 \%$. The above phenomenon shows that the heat dissipation performance of the 3-D orthogonal woven composite is not very good. This is closely related to the thermal conductivity capacity of each component in the composite structure - that is, the poor thermal conductivity of the component materials leads to the weak heat dissipation performance of the composite.

\subsection{The characteristic temperature distribution of each yarn system}

In order to clarify the heat transfer mechanism of the 3-D orthogonal woven composite under the non-uniform heat load, it is important to reveal the heat transfer process of each system from its specific structural characteristic.

The fabric reinforcement is the main load-carrying part of the composite, and the thermal conductivity capacity of the yarns is significantly higher than that of the resin, which is about four times that of the latter; therefore, exploring the temperature distribution characteristics of each part of the fabric reinforcement is of great significance for understanding the temperature-related structural effect of the 3-D orthogonal woven composite. As shown in Figure 5, the specific warp, weft, and Z-yarns that are closest to the directly heat load point are selected, and the temperature distribution curves along the length of each yarn are given.

It can be found that the temperature distribution on each yarn system has the obvious spatial morphology characteristics; that is, the temperature distribution along the length direction of each yarn is perceptibly affected by its spatial arrangement. In particular, this phenomenon is more pronounced on the Z-yarns. For the investigated specific warp and weft yarns, since the central region of each yarn is closest to the directly heat load area, the temperature is the highest. As mentioned above, the temperature on the weft yarn is slightly higher than that on the warp yarn. Due to the limitation of the thermal conductivity 


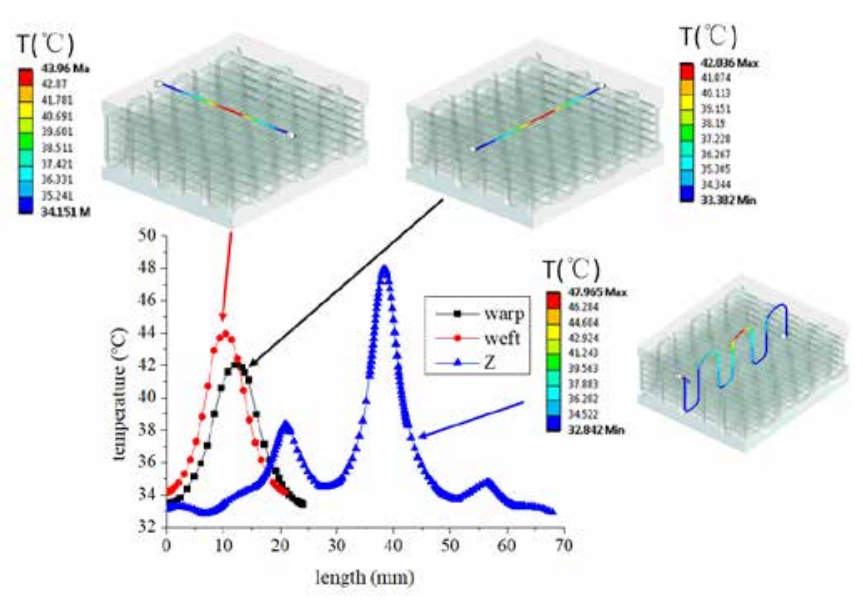

Figure 5. The temperature curves along the length direction of each yarn.

capacity of the yarns, the temperature drops sharply at both ends. For the specific Z-yarn, it has the highest temperature.

\section{Conclusions}

The thermodynamic behavior of 3-D orthogonal woven composite is studied to explore its structural heat transfer mechanism in a non-uniform heat load field based on FEA. By finite element modeling and analysis as well as considering the micro-structures of warp yarns, weft yarns, Z-yarns, and resin, the following conclusions have been obtained.

(1) The temperature on the surface of the resin is the highest due to the direct action area of the heat load. Furthermore, the Z-yarn system has the highest temperature, which is followed by the weft system, and then the warp system. This result is closely related to the structural characteristics of the 3-D orthogonal woven fabric reinforcement of the composite.

(2) For the two directions of transverse warp and weft, the temperature at the center is the highest, and it drops sharply in the two directions to the edge. The temperature drops from the highest at the center, where it is in the range of about $74^{\circ}-33^{\circ} \mathrm{C}$; the drop is approximately $55 \%$. Similarly, in the thickness direction, the temperature at the starting point is the highest, and it drops sharply along the thickness direction, with a drop of approximately 55\%. The above phenomenon shows that the heat dissipation performance of the 3-D orthogonal woven composite is not very good. This is closely related to the thermal conductivity capacity of each component in the composite structure.

(3) The temperature distribution on each yarn has the obvious spatial morphology characteristics of the corresponding yarn. In particular, this phenomenon is more pronounced on the Z-yarns. Moreover, due to the limitation of the thermal conductivity capacity of the yarns, the temperature drops sharply at both ends.
(4) For the prospects on the FEA-based thermodynamic analysis of the fiber-reinforced composite materials, the optimization design of composite materials resistant to the thermal damage can be focused on. It mainly includes the optimization for the raw materials of fibers and resin, the structural design of the reinforcement, and the fibers' orientation. In addition, in order to ensure that the designed and manufactured composite materials have the sufficient thermodynamic properties and meet the requirements of the practical applications, the conservative calculations of FEA must be applied.

\section{FUNDING}

This work is financially supported by the Open Project Program (No. YGKF202014) and Scientific Research Fund (No. ygy2002) of Yancheng Polytechnic College.

\section{ACKNOWLEDGMENTS}

The authors also gratefully acknowledge the financial supports from the High Education Science Foundation (No. 21KJB540007), the Natural Science Foundation (No. BK20201216) and Qinglan Project (No. Jiangsu Teacher 20193) of Jiangsu Province, the Integration Platform of Industry and Education of Jiangsu Higher Vocational Education (No. Jiangsu Vocational Education 2019-26), the Jiaxing Program of Science and Technology (No. 2019AD32001), and Open Project Program of Key Laboratory of Yarn Materials Forming and Composite Processing Technology of Zhejiang Province (No.MTC2021-02).

\section{References}

[1] Hu, J. (2008). 3-D fibrous assemblies: Properties, applications and modelling of three-dimensional textile structures. Woodhead Publishing Limited (Cambridge), pp. 1-32.

[2] Tong, L., Mouritz, A. P., Bannister, M. K. (2002). 3D fibre reinforced polymer composites. Elsevier Science Ltd (Oxford), pp. 1-12.

[3] Mouritz, A. P., Bannister, M. K., Falzon, P. J., Leong, K. $H$. (1999). Review of applications for advanced threedimensional fibre textile composites. Composites Part $A$, 30(12), 1445-1461.

[4] Miravete, A. (1999). 3-D textile reinforcements in composite materials. Woodhead Publishing Limited (Cambridge), 9-65.

[5] Hu, Q., Zhang, Y., Mao, Y., Memon, H., Qiu, Y., et al. (2019). A comparative study on interlaminar properties of $L$-shaped two-dimensional (2D) and three-dimensional (3D) woven composites. Applied Composite Materials, 26, 723-744.

[6] Hu, Q., Memon, H., Qiu, Y., Liu, W., Wei, Y. (2020). A comprehensive study on the mechanical properties of different $3 D$ woven carbon fiber-epoxy composites. Materials, 13(12), 2765.

[7] Rocha, I. B. C. M., van.der Meer, F. P., Raijmaekers, S., Lahuerta, F., Nijssen, R. P. L., et al. (2019). A combined experimental/numerical investigation on hygrothermal aging of fiber-reinforced composites. European Journal of Mechanics - A/Solids, 73, 407-419. 
[8] Liu, S., Zhang, J., Shim, B., Wang, L., Gu, B., et al. (2019). Damage and failure mechanism of $3 D$ carbon fiberlepoxy braided composites after thermo-oxidative ageing under transverse impact compression. Composites Part B: Engineering, 161, 677-690.

[9] Qin, G., Na, J., Mu, W., Tan, W., Yang, J., et al. (2018). Effect of continuous high temperature exposure on the adhesive strength of epoxy adhesive, CFRP and adhesively bonded CFRP-aluminum alloy joints. Composites Part B: Engineering, 154, 43-55.

[10] Gigliotti, M., Minervino, M., Lafarie-Frenot, M. C. (2016). Assessment of thermo-oxidative induced chemical strain by inverse analysis of shrinkage profiles in unidirectional composites. Composite Structures, 157, 320-336.

[11] Wang, M., Cao, M., Wang, H., Siddique, A., Gu, B., et al. (2017). Drop-weight impact behaviors of 3-D angle interlock woven composites after thermal oxidative aging. Composite Structures, 166, 239-255.

[12] Zhang, M., Sun, B., Gu, B. (2018). Experimental and numerical analyses of matrix shrinkage and compressive behavior of 3-D braided composite under thermo-oxidative ageing conditions. Composite Structures, 204, 320-332.
[13] Song, L., Li, J. (2012). Effects of heat accelerated aging on tensile strength of three dimensional braided/epoxy resin composites. Polymer Composites, 33(9), 1635-1643.

[14] Goo, N. S., Woo, K. (2003). Measurement and prediction of effective thermal conductivity for woven fabric composites. International Journal of Modern Physics B, 17(8n9):18081813.

[15] Siddiqui, M. O. R., Sun, D. (2013). Finite element analysis of thermal conductivity and thermal resistance behaviour of woven fabric. Computational Materials Science, 75(12), 45-51.

[16] Cheng L. (2010). Thermal expansion coefficients of carbon/epoxy braided composites. Journal of Solid Rocket Technology, 33(1), 108-111.

[17] Mohajerjasbi, S. (1997) Predictions for coefficients of thermal expansion of three-dimensional braided composites. AIAA Journal, 35(1), 141-144.

[18] Wang, H., Cao, M., Siddique, A., Sun, B., Gu, B. (2017). Numerical analysis of thermal expansion behaviors and interfacial thermal stress of $3 D$ braided composite materials. Computational Materials Science, 138, 77-91. 